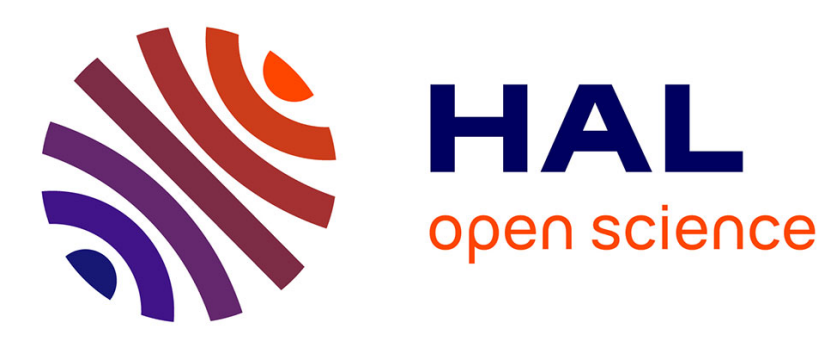

\title{
Riblets Induced Drag Reduction on a Spatially Developing Turbulent Boundary Layer
}

\author{
A. Bannier, E. Garnier, P. Sagaut
}

\section{To cite this version:}

A. Bannier, E. Garnier, P. Sagaut. Riblets Induced Drag Reduction on a Spatially Developing Turbulent Boundary Layer. Progress in Wall Turbulence: Understanding and Modelling, Jun 2014, LILLE, France. hal-01087636

\author{
HAL Id: hal-01087636 \\ https://hal.science/hal-01087636
}

Submitted on 26 Nov 2014

HAL is a multi-disciplinary open access archive for the deposit and dissemination of scientific research documents, whether they are published or not. The documents may come from teaching and research institutions in France or abroad, or from public or private research centers.
L'archive ouverte pluridisciplinaire HAL, est destinée au dépôt et à la diffusion de documents scientifiques de niveau recherche, publiés ou non, émanant des établissements d'enseignement et de recherche français ou étrangers, des laboratoires publics ou privés. 


\title{
Riblets Induced Drag Reduction on a Spatially Developing Turbulent Boundary Layer
}

\author{
Amaury Bannier, Eric Garnier and Pierre Sagaut
}

\begin{abstract}
Large eddy simulations have been conducted to gain further insight into the drag-reducing mechanisms of riblets in zero-pressure-gradient turbulent boundary-layer. The retained groove geometry achieves $9.8 \%$ drag-reduction on the controlled zone developing from $\operatorname{Re}_{\theta}=670$ to 975 . It is shown that the turbulent contribution to the drag - as defined by Fukagata et al. in [7] - is the most affected. In the light of the obtained results, energy and enstrophy budgets will finally conduct to isolate a key mechanism involved in the riblets drag-reduction.
\end{abstract}

\section{Introduction}

Making grooves on a surface increases the wetted area and, counter-intuitively, may reduce the drag for turbulent flows [22]. For the last three decades, those socalled "riblets" have remained under active consideration. They have been studied through experiments [22, 2], numerical simulations [4, 9] and also flight tests [5]. In their oil channel facility, Bechert et al. [2] achieve $8.2 \%$ friction reduction with a technologically-feasible design. However, a lack of understanding persists regarding the underlying mechanisms.

Based on viscous analyses, Luchini et al. [14] observed that, above both a clean and a ribbed surfaces, the Stokes flows converge asymptotically to the same far-field solution, providing the appropriate relative position of the two walls. For a given riblet geometry, one can thus compute the "virtual origin" $h$ at which a flat plate should be located to produce asymptotically the same Stokes flow. The length $h_{\|}$ can be defined for a longitudinal flow over the grooves (see figure 3 for illustration)

Amaury Bannier · Eric Garnier

ONERA - The French Aerospace Lab, Applied Aerodynamics Department, Meudon, France, email: amaury.bannier@onera.fr, eric.garnier@onera.fr

Pierre Sagaut

Universitée Pierre et Marie Curie - Paris 6, Institut Jean le Rond d'Alembert, Paris, France 
and, similarly, $h_{\perp}$ for the transverse Stokes flow above the same riblets. Luchini's "protrusion height" is defined as the difference between the two obtained virtual origins: $\Delta h=h_{\perp}-h_{\|}$. They describe this quantity as a measure of "how much the corrugated wall impedes the cross-flow more than it does the longitudinal flow".

García-Mayoral \& Jiménez [8] identified drag-reduction regimes, gathering in one previously proposed theories $[14,4,9,10]$. For low Reynolds number or small riblets, effects are well-characterized by $\Delta h$. When size or Reynolds increases, break-down mechanisms, based on Kelvin-Helmotz instabilities, cancel out the beneficial viscous effect. In their analysis, García-Mayoral \& Jiménez provide a scaling law for designing riblets within the appropriate regime.

Although the drag reduction is well-characterized by a Stokes flow analogy in the first regime, the mechanism itself remains turbulent per se: riblets always increase drag in laminar flows [3].

To further understand the drag-reduction riblets engender, the present work will focus at identifying its cause. Fukagata, Iwamoto \& Kasagi (FIK) [7] presented a decomposition of the skin friction. In their formulation, they split the contribution to the drag into different terms according to their physical origin. The drag-producing mechanisms can be isolated and their intensities spatially observed along the boundary layer.

After having described the retained riblets geometry and computational parameters next section, the FIK's decomposition will constitute the starting point of the present analysis $\S 3$. Each of the "bulk" and the turbulent contributions to the drag will be analyzed in $\S 4$ and $\S 5$. The observation of turbulent energy $\S 6$ and enstrophy $\S 7$ budgets will point out mechanisms responsible for the drag-reduction.

\section{Flow Configuration and Riblets Geometry}

Throughout this paper, the longitudinal, normal and spanwise directions will be respectively denoted $x, y$, and $z . U_{0}, \rho$ and $v$ stand for the reference fluid velocity, density and kinematic viscosity. The superscript + denotes quantity scaled with the friction velocity $u_{\tau}=\sqrt{\tau_{w} / \rho}$ and with $v$, where $\tau_{w}$ is the wall shear stress. The overbar is used for time averaged variables and Reynolds decomposition yields $f=\bar{f}+f^{\prime}$.

Under the constraint of realizable grooves, the most efficient riblets so far are the trapezoidal ones studied by Bechert et al. [2]. They have been shown to achieve up to $8.2 \%$ drag reduction. The rib angle is $\alpha=30^{\circ}$ and the height-over-width ratio is fixed to $h_{r i b} / s_{r i b}=0.5$. This riblets design leads to a "protrusion height" of $\Delta h \approx 0.10 s_{r i b}=0.20 h_{r i b}$, a value which can be compared to the optimal limit $\Delta h_{\max } \approx 0.13 s_{\text {rib }}$ computed analytically by Luchni et al. [14]. Further increasing our $\Delta h$ requires to sharpen the riblets even more and would lead to technologically unfeasible design. The retained geometry is depicted in figure 1. 
Regarding the sizing of the groove, García-Mayoral \& Jiménez [8] provide a simplified stability model aiming at estimated the optimal riblets dimension for a given geometry. With our design, $h_{r i b}$ has to be close to approximately 8 wall-units.

The botoom surface of the computational domain is not entirely covered by riblets. Smooth surfaces are placed up- and down-stream of the controlled zone. The question of the relative vertical position of the riblets with respect to those flat plates has to be addressed. We set the ordinate origin $y=0$ at the flat wall location and define $\Delta y_{0}$ the (likely negative) vertical position of the ribs valley ( $c f$ figure 3 right-hand-side axis). Among other possibilities, ribets can be set such as either the valleys or the tips are located at the flat plate level, corresponding respectively to $\Delta y_{0}=0$ and $\Delta y_{0}=-h_{r i b}$. Alternatively, the use of the previously defined virtual origin $h_{\|}$is a consistent choice with respect to viscous effects. Here, decision is made to cancel, or at least restrict, the net pressure force acting at the emergence and at the disappearance of the grooves. The riblets relative position is set to $\Delta y_{0}=-\frac{h^{2}}{s} \tan \left(\frac{\alpha}{2}\right) \approx-0.13 h_{\text {rib }}$ so as to preserve the same cross-sectional area.

The simulation is conducted in a zero-pressure gradient turbulent boundary layer (ZPGTBL). The Reynolds number based on the momentum thickness develops from $R e_{\theta}=670$, at the beginning of the grooved area to $R e_{\theta}=925$. The corresponding friction Reynolds number ranges from $R e_{\tau}=260$ to $R e_{\tau}=350$. The Synthetic eddy method (SEM) [16] is used to generate the turbulent inflow. Flow properties at the beginning of the work area are successfully compared to reference DNS [20, 19].

The ONERA in-house flow solver FUNk which has been used, for which details and validations can be found in $[15,11]$, resolves the Direct Navier-Stokes (DNS) and the Large Eddy Simulations (LES) equations for compressible flows on multiblock structured grids. The simulation Mach number is set to 0.2. In this condition, density and viscosity fluctuations are negligible and the flow will be considered incompressible. The explicit selective mixed scale model [13] is used to compute the subgrid viscosity for LES simulations.

The tiny size of the riblets strongly constrains the cells dimensions. In order to accurately reproduce their shape, $\Delta y^{+}$and $\Delta z^{+}$are maintained around 1 close to the wall. $\Delta x^{+}$is set to 25 . Such a resolution goes beyond state-of-the-art DNS baseline refinements in the normal and spanwise directions. The longitudinal resolution justifies the qualification of LES and the use of a subgrid-scale model. The size of the computational domain is $38 \delta_{0} \times 10 \delta_{0} \times 3.3 \delta_{0}$, where $\delta_{0}$ denotes the inflow boundary layer thickness. Two-point correlations have been computed to ensure the domain is wide enough. The computational domain counts a total of $413 \times 143 \times 673$ cells. 


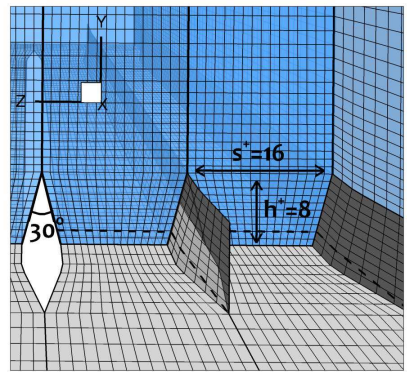

Fig. 1 Riblets geometry and mesh representation.

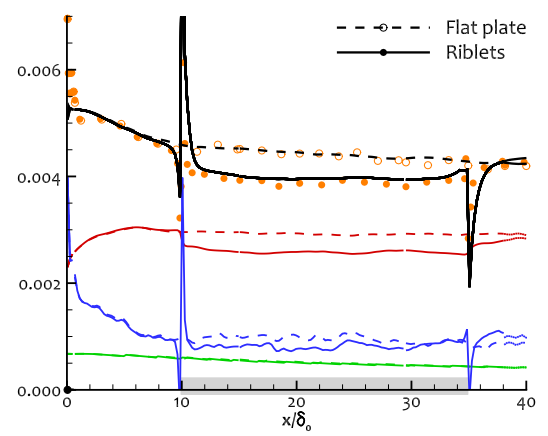

Fig. 2 Skin friction coefficient and FIK decomposition: $c_{f}$ from numerical flux (black); $c_{f, 1}$ (green); $c_{f, 2}$ (red); $c_{f, 3}$ (blue); $c_{f, 1}+c_{f, 2}+c_{f, 3}$ (symbols).

\section{Analysis of the Wall Friction}

The time- and spatial-averaged wall friction coefficient in figure 2 is defined by $c_{f}=\frac{1}{L_{z}} \int_{z}\left(\bar{\tau}_{w} / \frac{1}{2} \rho U_{0}^{2}\right) \mathrm{d} z$. Over the ribbed plate, the spanwise averaging has to be changed into $\frac{1}{L_{z}} \int_{\tilde{L}_{z}} \cdot \mathrm{d} l$, where $\tilde{L}_{z}$ denotes the wetted span at the riblets surface and $L_{z}$ the corresponding span of the flat plate.

Upstream the ribbed zone, the brief transitory burst of $c_{f}$ is due to the sudden geometric outbreak of riblets out the planes. The performance loss caused by riblets appearance is approximately overcome by the afterward persistent benefit. Taking into account these up- and down-stream effects, the global drag reduction adds up to $9.8 \%$ of the baseline drag on the controlled domain. The pressure drag, due to the 3-dimensional shape of the riblets leading and trailing edges, is found to be negligible, justifying the previously discussed choice of riblets-versus-plate relative vertical position.

The FIK's decomposition is applied here. Using the notation of Deck et al. [6], the friction coefficient is split as $c_{f}=c_{f, 1}+c_{f, 2}+c_{f, 3}$. Because of the geometric complexity of our flow, the original FIK's formulation cannot be applied as is. Following Peet and Sagaut [17] who derived the equivalent of FIK's channel flow decomposition for three-dimensional complex wall shape, a similar formula can be obtained from FIK's boundary layer formulation:

$$
\begin{aligned}
& c_{f, 1}(x)=\frac{4}{\alpha \operatorname{Re} \delta} \iint_{\Sigma(x)} \frac{\bar{u}_{x}}{U_{0}} \frac{\mathrm{d} \Sigma}{L_{z} \delta} \\
& c_{f, 2}(x)=\frac{4}{\alpha} \iint_{\Sigma(x)}\left(1-\frac{y}{\delta}\right) \frac{-\overline{u^{\prime} v^{\prime}}}{U_{0}^{2}} \frac{\mathrm{d} \Sigma}{L_{z} \delta} \\
& c_{f, 3}(x)=-\frac{4}{\alpha} \iint_{\Sigma(x)} \frac{1}{2}\left(1-\frac{y}{\delta}\right)^{2} \frac{\delta}{\rho U_{0}^{2}} \overline{\left(\frac{\partial \rho u_{x}}{\partial t}+I_{x}+\frac{\partial p}{\partial x}\right)} \frac{\mathrm{d} \Sigma}{L_{z} \delta}
\end{aligned}
$$




$$
\begin{aligned}
& \alpha(x)=1+\int_{\tilde{L}_{z}(x)} \frac{y}{\delta}\left(\frac{y}{\delta}-2\right) \frac{\mu \partial \bar{u}_{x} / \partial n}{F_{w}} \frac{\mathrm{d} l}{L_{z}} \\
& I_{x}(x)=\rho \bar{u}_{i} \frac{\partial \bar{u}_{x}}{\partial x_{i}}-\mu \frac{\partial^{2} \bar{u}_{x}}{\partial x^{2}}+\rho \frac{\partial \overline{u_{x}^{\prime} u_{x}^{\prime}}}{\partial x}
\end{aligned}
$$

where $\Sigma(x)$ stands for the transverse plane at constant $x$ between the wall and the boundary layer height. The coefficient $\alpha$, which takes into account the wall stress distribution along the normal direction, is close to unity as soon as $h_{r i b}^{+} / R e_{\tau}$ is negligible. For the present simulation, $1-\alpha$ is of the order of $3 \%$.

In figure 2 , the "bulk" term $c_{f, 1}$ is largely unaffected by the riblets. This term is related to the mean velocity $\bar{u}_{x}$ which is examined in the following section. The second term, $c_{f, 2}$, accounting for the turbulent contribution to the drag, is the most affected by the riblets presence. However, ratios $c_{f, 2} / c_{f}$ for both the flat and the ribbed cases perfectly collapse, supporting a scaling in wall units of the turbulence mechanisms. Turbulence quantities will be investigated $\S 5$. Finally, term $c_{f, 3}$ is associated with the streamwise growth of the boundary layer. In the presence of riblets, both the term itself and its relative contribution in $c_{f}$ are reduced. Although the evolution of this last term is not well understood yet, it has to satisfy $\left(c_{f, 1}+c_{f, 3}\right) / c_{f}=1-\left(c_{f, 2} / c_{f}\right)$ to fulfill previous requirements on $c_{f, 1}$ and $c_{f, 2}$.

\section{Mean Velocity Field}

The "bulk" term $c_{f, 1}$ is directly linked to the mean streamwise velocity and is proportional to the mean flow rate within the boundary layer.

Before depicted the mean velocity profile, the question of the vertical ordinate origin must be clarified. The vertical origin $y=0$ in figures still corresponds to the smooth wall location. However, for the ribbed wall, at what location $\Delta y$ should the riblets valley be located? A choice $\Delta y_{0}$ has already been made $\S 2$ to answer this question regarding the geometrical set up. However, to ease the comparison with the smooth wall flow, another ordinate origin $\Delta y_{(u)}$ may be more relevant to plot the mean flow profile $\bar{u}_{x}$. Among the different options listed in section 2, the choice based on the longitudinal Stokes flow, leading to $\Delta y_{(u)}=-h_{\|} \approx-0.63 h_{r i b}$, appears to be the most pertinent. Indeed, since riblets are quasi-immersed in a viscous sublayer, the mean flow field is expected to be very similar to the Stokes flow solution. Figure 3 illustrates this point. From the riblets floor up to some few wall-units above the tips, the turbulent and the Stokes viscous profile closely fit.

Since the mean velocity depends on $z$ nearby the riblets, spanwise-averaging of the velocity is performed to allow an intelligible comparison. Two different scalings are used for the non-dimensionalization in figure 4 . The first one is based upon the actual wall friction velocity $u_{\tau}=\sqrt{c_{f} / \rho}$. This scaling is used to compare terms in usual wall units and highlight variables whose magnitude is proportional to the wall shear stress. Alternatively, to assess the absolute evolution with respect to the 


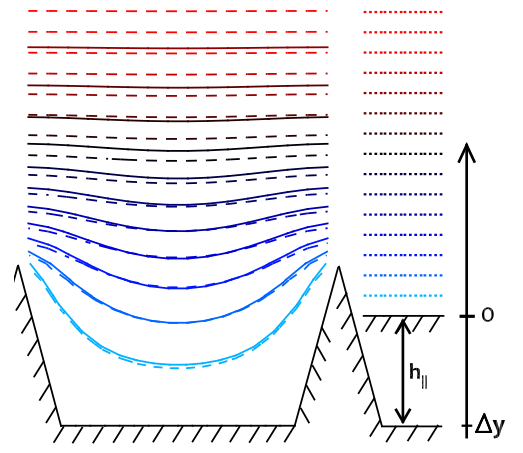

Fig. 3 Transverse view of mean streamwise velocity iso-contours over riblets: LES turbulent flow at $R e_{\theta}=750$ (solid); Stokes flow (dashed). Right-sided dotted iso-contours denotes the Stokes flow over a flat plate located located $h_{\|}$above riblets valleys.

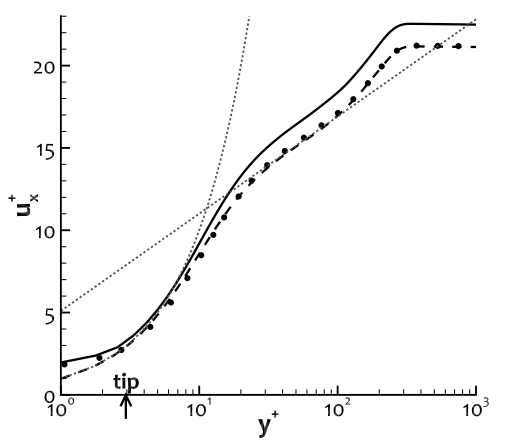

Fig. 4 Mean spanwise-averaged streamwise velocity profile at $R e_{\theta}=750$ over flat plate (dash) and riblets in actual (solid) and nominal (symbols) wall-units. Linear $\left(u^{+}=y^{+}\right)$ and logarithmic $\left(u^{+}=\log \left(y^{+}\right) / 0.39+5.1\right)$ profiles (dotted). The arrow indicates the riblets tip location.

uncontrolled - smooth wall - case, another scaling is used, based on the friction velocity $u_{\tau}^{\text {nominal }}$ of the baseline clean plate.

Above the flat surface, the velocity profile is fitted by the log-law with coefficients $\kappa=0.39$ and $C^{+}=5.1$. Riblets cause a thickening of the viscous layer and a significant upward shift of the log-law [1]: $\Delta u^{+}=\sqrt{2 / c_{f}^{\text {rib }}}-\sqrt{2 / c_{f}^{\text {clean }}} \approx 1.14$.

The nominal friction velocity scaling highlights the minor impact of the riblets on $\bar{u}_{x}$ outside the viscous sublayer: the two velocity profiles neatly collapse above $y^{+} \approx 10$. This very spatially-limited modification of the mean velocity explains why riblets do not significantly impact the "bulk" coefficient $c_{f, 1}$. Nevertheless, since the global $c_{f}$ is reduced in presence of riblets, the relative contribution of $c_{f, 1}$ is increased.

Conversely to $c_{f, 1}$, the relative turbulent contribution to the drag $c_{f, 2} / c_{f}$ remains unchanged by the riblets, while the absolute value for $c_{f, 2}$ decreases. The impact of riblets on the turbulence properties is investigated in the following sections.

\section{Turbulent Statistics}

The root-mean-square turbulent velocity fluctuations and the normal Reynolds stress $\overline{u^{\prime} v^{\prime}}$ are displayed in Figure 5. On the whole, all intensities are reduced in absolute term (i.e. scaled using nominal wall friction velocity), whereas, modifications are much limited in (actual) wall-unit scaling. More specifically, the $u_{r m s}$ profile is only affected by the riblets below $y^{+}=17$. This results in a slight lowering and upward shift of its maximum peak. The profiles for $v_{r m s}$ and $\overline{u^{\prime} v^{\prime}}$ above the ribbed and 
smooth walls collapse almost perfectly, consistently with their close role with the wall friction generation. Finally, $w_{r m s}$ is somewhat increased above $y^{+}=10$.

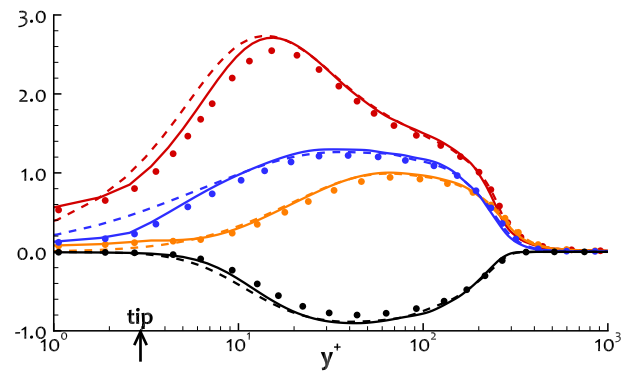

Fig. 5 Rms turbulent intensities $u_{r m s}^{+}$(red), $v_{r m s}^{+}$(orange), $w_{r m s}^{+}$(blue) and Reynolds normal stress $\overline{u^{\prime} v^{\prime}}$ (black), at $R e_{\theta}=750$, over flat plate (dash) and riblets in actual (solid) and nominal (symbols) wall-units. The arrow indicates the riblets tip location.

Note that only the spanwise component $w_{r m s}$ is damped in the riblets vicinity, in agreement with Luchini's vision of riblets as device impeding selectively the transverse over the longitudinal flow [14].

Because $c_{f}=2\left(U_{0}^{+}\right)^{-2}$, the ratio $c_{f, 2} / c_{f}$ explicitly reveals a weighted integral of $\overline{u^{\prime} v^{\prime}}$, scaled in wall units. Owing to the negligible impact of riblets on the wallunit-scaled profile for the Reynolds normal shear stress, the turbulent contribution $c_{f, 2}$ and the drag coefficient $c_{f}$ are de facto proportional.

\section{Budget of Turbulent Kinetic Energy}

The impact of riblets on turbulence is now investigated. It has been shown in $\S 5$ that, in overall, fluctuations intensities scale in wall-units.

The same scaling will be adapted for energy budget, and beyond that, most terms intensities are essentially unchanged by riblets, assuming the appropriate vertical origin ordinate is chosen. The previously used Stokes virtual origin $\Delta y_{(u)}=-h_{\|}$ does not lead, here, to a meaningful collapse of the curves. However, a striking superimposition is observed if one shifts the riblets further approximately one wallunits downward. This coordinate shift brings back to the velocity shift of the log layer $\left(\Delta u^{+} \approx 1\right)$ computed in $\S 4$. The ordinate origin $\Delta y_{(K)}^{+}=\Delta y_{(u)}^{+}-\Delta u^{+} \approx$ $-0.75 h_{\text {rib }}^{+}$allows to make the log-law region of both flows starts at the same location. This positioning will be used for the following energy budgets of this article.

The transport equation for the turbulent kinetic energy $k=\frac{1}{2} \overline{u_{i}^{\prime} u_{i}^{\prime}}$ reads:

$$
\frac{\partial k}{\partial t}=\underbrace{-\bar{u}_{i} \frac{\partial k}{\partial x_{i}}}_{1}-\underbrace{2 v s_{i j} \frac{\partial u_{i}^{\prime}}{\partial x_{j}}}_{2}-\underbrace{\overline{u_{i}^{\prime} u_{j}^{\prime}} \frac{\partial \bar{u}_{j}}{\partial x_{i}}}_{3}-\frac{\partial}{\partial x_{i}}(\underbrace{\frac{1}{\rho} \overline{p^{\prime} u_{i}^{\prime}}}_{4}-\underbrace{2 v \overline{s_{i j} u_{j}^{\prime}}}_{5}+\underbrace{\overline{u_{i}^{\prime} u_{j}^{\prime} u_{j}^{\prime}}}_{6})
$$




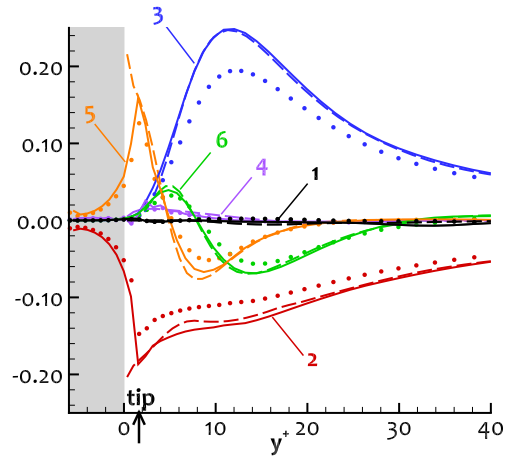

Fig. 6 Profiles for the turbulent kinetic energy budget terms, at $R e_{\theta}=750$, over flat plate (dash) and riblets in actual (solid) and nominal (symbols) wall-units. The arrow indicates the riblets tip location.

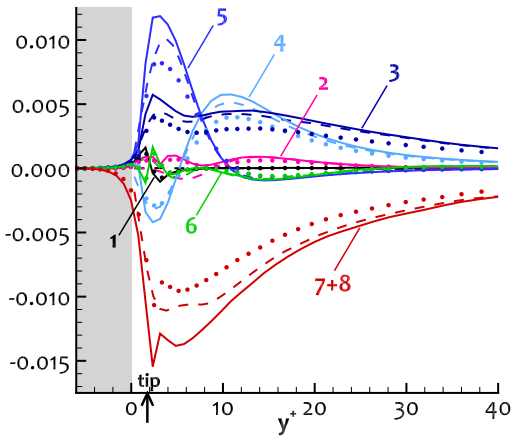

Fig. 7 Profiles for the turbulent enstrophy budget terms. For line legend, refer to figure 6 aside.

where Einstein implicit summation is used. The wall-normal distributions of those terms are shown in figure 6.

Terms 1 and 4, standing for the transport work done by the total dynamic pressure, have little impacts on the budget. The production term 3 and the turbulent diffusion term 6 exhibit profiles almost identical between flat and ribbed plates. The main difference between flows is an enhancement of the dissipation (term 2) in the $y^{+} \in[6,12]$ layer. To balance the budget, a tiny downward shift of the production level (term 3$)$ in the viscous sublayer $\left(y^{+}<6\right)$ provides the additional energy. The viscous diffusion term 5 ensures the appropriate energy transport between those two zones.

Compare to the smooth wall case in wall-units, more energy is pumped from the mean flow to be dissipated into heat. In absolute scaling however, dissipation levels are greatly reduced. Thus, the key-role in the riblets drag-reducing mechanisms seems to be played by the dissipation term 2 . It can be rewritten into:

$$
2 v \overline{s_{i j} \frac{\partial}{\partial x_{j}} u_{i}^{\prime}}=v \overline{\omega_{i}^{\prime} \omega_{i}^{\prime}}+2 v \frac{\partial^{2}}{\partial x_{i} \partial x_{j}} \overline{u_{i}^{\prime} u_{j}^{\prime}},
$$

with $\omega_{i}$ the flow vorticity components. The first term, called turbulent enstrophy, is observed to be the predominant one [21]. The turbulent dissipation is therefore directly related to the turbulent enstrophy. Its budget is now broken up.

\section{Budget of Turbulent Enstrophy}

The detailed equation for the enstrophy budget of an incompressible flow can be found in [21], eq. (3.3.38). Labels in figure 7 correspond to following terms. Term 
1 accounts for the convection by the mean flow. Terms 2 through 5 are production terms: term 2 represents the gradient production and is the enstrophy analogue for the kinetic energy budget term 3 in equation (6). Terms 3 and 4 denote the production (or removal) caused by the stretching of vorticity fluctuations by the turbulent and the mean strain rate. Term 5 is a mixed production term also appearing in the budget for $\frac{1}{2} \bar{\omega}_{i} \bar{\omega}_{i}$. Terms 6 and 7 represent the turbulent and the viscous diffusion. Finally, term 8 express the enstrophy dissipation. Terms 7 and 8 , both accounting for viscous effects, have been gathered for clarity's sake in figure 7 .

The main effects of riblets is to enhance most of the terms in the enstrophy budget. More specifically, a striking alteration occurs with the production term 2, whose expression is : $\overline{u_{j}^{\prime} \omega_{i}^{\prime}}\left(\partial \overline{\omega_{i}} / \partial x_{j}\right)$. It accounts for the transfer between the mean and the turbulent squared vorticity. Term 2 acts as a sink term below $y^{+}=9$ for the smooth wall, but becomes a production term for ribbed wall. This change is due to the creation, by the riblets, of mean vorticity in the normal direction $\bar{\omega}_{y}$ in order to satisfy the non-slip condition on grooves quasi-vertical sides. By producing mean normal vorticity, riblets induce extra production in the enstrophy budget - production which is counter-balanced by viscous effect - and enhance the enstrophy level. Since enstrophy translates into turbulent kinetic energy dissipation, the turbulent activity drops because of the increased dissipative nature of the flow. This drop explains the absolute reduction of all turbulence intensities, figure 5. A new equilibrium establishes with a weaken turbulence activities. All levels of the turbulent budget (6) are dumped in absolute terms, including the dissipation which has been initially enhanced. As long as the riblets maintain an important level of enstrophy production, the flow budget remains balanced with small turbulence levels.

The reduce level of turbulence intensities, and especially the one for the normal Reynolds shear stress, involves a drop of the momentum diffusion towards the wall and a fall of the wall friction. Similar drag mechanisms are observed as well for other drag reducing devices, such as transverse wall oscillation [18] and suboptimal control theory [12].

\section{Outlook}

Skin friction has been decomposed and the turbulent contribution was shown to be the most affected by the riblets. The modification of turbulence properties was investigated through a dissection of the budgets of the turbulent kinetic energy first, and of the main part of its dissipation - the enstrophy - thereafter. The mean normal vorticy created on riblets lateral sides turns out to be behind an extra dissipation of the turbulence intensities, which eventually reduces the drag.

Further investigations of the energetic exchanges involved in the turbulence above riblets, and, more especially, within the transient area downstream the forefront of the ribbed wall, may bring further details and help clarifying the dragreduction mechanisms. 
Acknowledgements This work was granted access to the HPC resources from IDRIS under the allocation 2014-100392 made by the GENCI. The thesis of Amaury Bannier is partly funded by Ecole Polytechnique.

\section{References}

1. Aupoix, B., Pailhas, G., Houdeville, R.: Towards a general strategy to model riblet effects. AIAA J. 50(3), 708-716 (2012)

2. Bechert, D.W., Bruse, M., Hage, W., Van Der Hoeven, J.G.T., Hoppe, G.: Experiments on drag-reducing surfaces and their optimization with an adjustable geometry. J. Fluid Mech. 338, 59-87 (1997)

3. Choi, H., Moin, P., Kim, J.: On the effect of riblets in fully developed laminar channel flows. Phys. Fluids A 3(8), 1892-1896 (1991)

4. Choi, H., Moin, P., Kim, J.: Direct numerical simulation of turbulent flow over riblets. J. Fluid Mech. 255, 503-539 (1993)

5. Coustols, E., Savill, A.: Turbulent skin-friction drag reduction by active and passive means: parts 1 and 2. Special course on skin-friction drag reduction, Brussels, Belgium (1992)

6. Deck, S., Renard, N., Laraufie, R., Weiss, P.E.: Large-scale contribution to mean wall shear stress in high-reynolds-number flat-plate boundary layers up to $R e_{\delta}=13650$. J. Fluid Mech. 743, 202-248 (2014)

7. Fukagata, K., Iwamoto, K., Kasagi, N.: Contribution of reynolds stress distribution to the skin friction in wall-bounded flows. Phys. Fluids 14(11), L73 (2002)

8. García-Mayoral, R., Jiménez, J.: Drag reduction by riblets. Philos. Trans. R. Soc. A 369(1940), 1412-1427 (2011)

9. Goldstein, D.B., Tuan, T.C.: Secondary flow induced by riblets. J. Fluid Mech. 363, 115-151 (1998)

10. Jiménez, J.: Turbulent flows over rough walls. Annu. Rev. Fluid Mech. 36, 173-196 (2004)

11. Larchevêque, L., Sagaut, P., Lê, T.H., Comte, P.: Large-eddy simulation of a compressible flow in a three-dimensional open cavity at high reynolds number. J. Fluid Mech. 516, 265301 (2004)

12. Lee, C., Kim, J., Choi, H.: Suboptimal control of turbulent channel flow for drag reduction. J. Fluid Mech. 358, 245-258 (1998)

13. Lenormand, E., Sagaut, P., Ta Phuoc, L., Comte, P.: Subgrid-scale models for large-eddy simulation of compressible wall bounded flows. AIAA J. 38(8), 1340-1350 (2000)

14. Luchini, P., Manzo, F., Pozzi, A.: Resistance of a grooved surface to parallel flow and crossflow. J. Fluid Mech. 228, 87-109 (1991)

15. Mary, I., Sagaut, P.: Large eddy simulation of flow around an airfoil near stall. AIAA J. 40(6), 1139 (2002)

16. Pamiès, M., Weiss, P.E., Garnier, E., Deck, S., Sagaut, P.: Generation of synthetic turbulent inflow data for large-eddy simulation of spatially-evolving wall-bounded flows. Phys. Fluids 21(4), 045103 (2009)

17. Peet, Y., Sagaut, P.: Theoretical prediction of turbulent skin friction on geometrically complex surfaces. Phys. Fluids 21(10), 105,105 (2009)

18. Ricco, P., Ottonelli, C., Hasegawa, Y., Quadrio, M.: Changes in turbulent dissipation in a channel flow with oscillating walls. J. Fluid Mech. 700, 77-104 (2012)

19. Schlatter, P., Örlü, R.: Assessment of direct numerical simulation data of turbulent boundary layers. J. Fluid Mech. 659, 116-126 (2010)

20. Spalart, P.R.: Direct simulation of a turbulent boundary layer up to $\operatorname{Re}_{\theta}=1410$. J. Fluid Mech. 187, 61-98 (1988)

21. Tennekes, H., Lumley, J.L.: A first course in turbulence. MIT Press (1972)

22. Walsh, M.J., Weinstein, L.M.: Drag and heat transfer on surfaces with small longitudinal fins. AIAA Paper 78-1161 (1978) 